\title{
Epigenetic reprogramming promotes the antiviral action of IFNa in HBV-infected cells
}

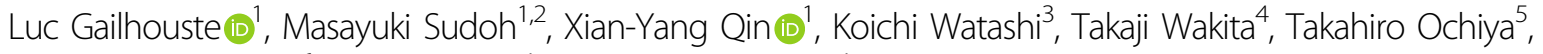 \\ Tomokazu Matsuura ${ }^{6}$, Soichi Kojima ${ }^{1}$ and Yutaka Furutani ${ }^{1}$
}

\begin{abstract}
Chronic hepatitis B virus (HBV) infections remain a health burden affecting 250 million people worldwide. Thus far, available interferon-alpha (IFNa)-based therapies have shown unsatisfactory cure rates, and alternative therapeutic molecules are still required. However, their development has been hampered because accessible cell models supporting relevant HBV replication and appropriate antiviral activity are lacking. Strategies that reverse epigenetic alterations offer a unique opportunity for cell reprogramming, which is valuable for restoring altered cellular functions in human cell lines. This work aimed to investigate the feasibility of converting HepG2 cells that stably overexpress the HBV entry receptor (sodium/taurocholate cotransporting polypeptide, NTCP) toward IFNa-responsive cells using epigenetic reprogramming. Herein, we showed that an epigenetic regimen with non-cytotoxic doses of the demethylating compound 5-azacytidine restored the anti-HBV action of IFNa in epigenetically reprogrammed HepG2NTCP-C4 cells, named REP-HepG2-NTCP cells. Thus, a significant inhibition in HBV DNA levels was measured in REPHepG2-NTCP cells after IFNa treatment. This inhibitory effect was associated with the enhancement of IFNa-mediated induction of critical interferon-stimulated genes (ISGs), which was limited in non-reprogrammed cells. In particular, our data indicated that re-expression of 2'-5'-oligoadenylate synthetase 1 (OAS1) and interferon regulatory factor 9 (IRF9) was the result of an epigenetically driven unmasking of these genes in reprogrammed cells. At last, we evaluated the therapeutic potential of the IFN analog CDM-3008 in REP-HepG2-NTCP cells and demonstrated the efficiency of this chemical compound in triggering ISG induction and HBV inhibition. In summary, this study shows that epigenetic reprogramming promotes the IFNa response in HBV-infected cells and is potentially attractive for cell-based experimental screening of IFN-like compounds.
\end{abstract}

\section{Introduction}

Hepatitis B virus (HBV) infections represent a major public health issue. Despite effective vaccination, $\sim 250$ million individuals are chronically infected with the virus worldwide $^{1}$. Without treatment, chronic HBV infections gradually progress to liver fibrosis and cirrhosis $^{2}$. Liver

\footnotetext{
Correspondence: Luc Gailhouste (luc.gailhouste@riken.jp) or

Yutaka Furutani (yfurutani@riken.jp)

${ }^{1}$ Liver Cancer Prevention Research Unit, RIKEN Cluster for Pioneering Research, Wako, Japan

${ }^{2}$ Department of Translational Research, Joint Research Center for Human Retrovirus Infection, Kagoshima University, Kagoshima, Japan

Full list of author information is available at the end of the article Deceased: Soichi Kojima
}

Edited by I. Amelio cirrhosis is associated with an increased risk of hepatocellular carcinoma (HCC), one of the leading causes of cancer-related death ${ }^{3}$. Currently, HBV therapeutic strategies include two formulations of interferon-alpha (IFN $\alpha)$ and five nucleos(t)ide analogs ${ }^{4}$. Nucleos(t)ide analogs negatively modulate the viral polymerase and de novo synthesis of HBV DNA ${ }^{5}$, whereas IFNa interferes with viral replication by inducing multiple interferonstimulated genes (ISGs), subsequently affecting various steps of the viral life cycle ${ }^{6,7}$. However, more effective therapies are still required because of the relatively low curative rates obtained. Notably, prolonged treatments with IFN $\alpha$ are associated with side effects, and cessation of nucleos(t)ide analog treatments is commonly 
accompanied by a virological relapse due to the inability of these molecules to eradicate HBV covalently closed circular DNA, which accumulates in hepatocyte nuclei and functions as a persistent template for virus production $^{8}$.

Understanding the molecular mechanisms by which IFN $\alpha$ mediates its antiviral action is critical for the development of new potent IFN-like compounds. In addition to primary human hepatocytes (PHHs), various experimental cell culture systems have provided alternative and more accessible tools for the study of anti-HBV molecules $^{9-11}$. Thus far, additional efforts have been required to obtain more-consistent models that achieve HBV infection/replication rates and IFN-induced antiHBV responses similar to those observed in PHHs. HepG2 cells that stably overexpress the HBV entry receptor sodium/taurocholate cotransporting polypeptide $(N T C P)$, also known as solute carrier family 10 member 1 (SLC10A1), represent an affordable and wellcharacterized model widely used in HBV research laboratories ${ }^{12-14}$. However, low ISG induction levels and limited or inconsistent anti-HBV activities frequently preclude the use of HepG2-NTCP cells for reliable antiHBV drug-screening applications ${ }^{15}$. We previously reported that hepatic cell lines treated with low concentrations of the DNA-demethylating reagent 5azacytidine (5-AZA) recovered gene expression profiles and functional features similar to hepatocytes ${ }^{16,17}$. The present work was based on the hypothesis that this procedure, named epigenetic reprogramming, can restore a consistent antiviral response in HepG2-NTCP cells through the re-expression of epigenetically silenced ISGs. Here, our results showed restoration of the IFNa response in epigenetically reprogrammed HepG2-NTCP-C4 (REPHepG2-NTCP) cells, whereas IFN $\alpha$ had no effect in nonreprogrammed HepG2-NTCP-C4 cells. This IFNa response was correlated with an augmentation of major ISGs known to be associated with the antiviral effect of IFN $\alpha$ in patients. Methylation analysis revealed the epigenetic unmasking of critical ISGs in REP-HepG2-NTCP cells, which were silenced by hypermethylation before reprogramming. Finally, we evaluated the potential of epigenetically reprogrammed cells for new drug screening by assessing the anti-HBV action of an IFN-like chemical compound.

\section{Results}

Epigenetic reprogramming restores the IFNa response in HepG2-NTCP-C4 cells

To explore the hypothesis that a demethylating treatment could promote cell antiviral activity, we first compared global gene expression profiles between 5AZA-treated HepG2 cells and control HepG2 cells using microarray data from our previous work ${ }^{16}$. The results from a gene set enrichment analysis (GSEA) highlighted three signatures related to the immune response: (i) IFN $\alpha$ response, (ii) IFN $\gamma$ response, (iii) and complement system (Fig. 1A). In particular, among the 90 genes that composed the IFN $\alpha$ response gene set, 38 genes appeared significantly enriched in HepG2 cells after epigenetic reprogramming (Fig. 1B), supporting a possible improvement of the antiviral response in these cells.

Given the low susceptibly of the parental HepG2 cells for HBV infection, the HepG2-NTCP cell line was used in this study. In particular, we selected the HepG2NTCP-C4 clone for epigenetic reprogramming because HepG2-NTCP-C4 cells exhibited high NTCP expression levels and high susceptibility to HBV infection, as previously described by Iwamoto and colleagues ${ }^{13}$. Before reprogramming, we confirmed the high expression levels of NTCP in HepG2-NTCP-C4 cells compared with the parental HepG2 cells (Fig. 2A). Our previous studies reported efficient epigenetic reprogramming in various cell lines using low doses of $5-\mathrm{AZA}^{16-18}$. To determine the optimal concentration of the reagent for HepG2-NTCP-C4 epigenetic reprogramming, we analyzed cell viability after 5-AZA exposure. Our data showed a dose-response effect of the compound on cell growth, with marked cytotoxicity observed from $4 \mu \mathrm{M}$ (Fig. 2B). In contrast, no significant cell toxicity or morphological alterations were observed at concentrations up to $2 \mu \mathrm{M}$ after 5 days of treatment. Based on our previous work, a minimum of 7-10 days of treatment is usually essential to ensure sufficient demethylation and re-expression of epigenetically silenced genes in HCC cells using 5-AZA at non-cytotoxic doses ${ }^{17}$. Accordingly, we established a protocol for the epigenetic reprogramming of HepG2-NTCP-C4 cells based on a $2 \mu \mathrm{M} 5$-AZA regimen for 10 days with daily replacement of the medium.

To determine whether epigenetic reprogramming could stimulate IFN $\alpha$ antiviral activity, REP-HepG2-NTCP cells were infected with HBV using polyethylene glycol (PEG) 8000 at an MOI of 100 for $24 \mathrm{~h}$ after completing the reprogramming procedure, and HBV DNA levels were quantified following treatment with 1,000 and 10,000 IU/ $\mathrm{mL}$ IFN $\alpha$ for 9 days (Fig. 2C). Importantly, we confirmed that IFN $\alpha$ and 5-AZA combination showed no cytotoxic effect in the cells after 9 days of treatment (S. Figure 1A). As expected, IFN $\alpha$ did not affect non-reprogrammed HepG2-NTCP-C4 cells, as the levels of HBV DNA remained unchanged after treatment (Fig. 2D). In contrast, the number of HBV DNA copies was reduced by $57.38 \% \pm 0.09 \%$ and $63.97 \% \pm 0.07 \%$ in REP-HepG2NTCP cells in response to 1,000 and $10,000 \mathrm{IU} / \mathrm{mL}$ IFN $\alpha$, respectively $(p<0.0001, t$ test), arguing for efficient restoration of the anti-HBV effect of IFN $\alpha$ in reprogrammed cells. 
A

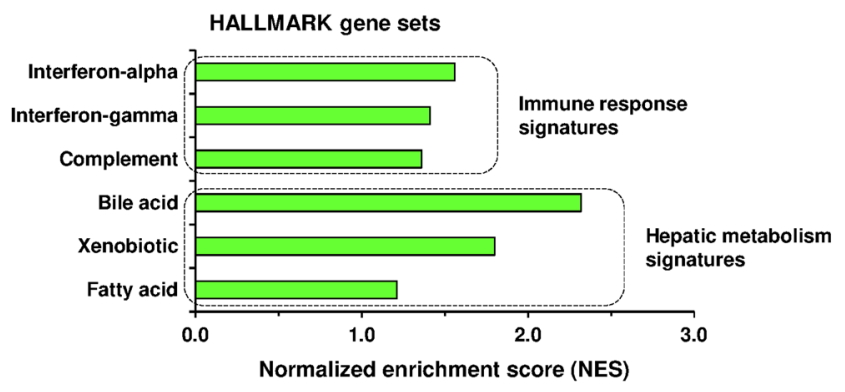

B
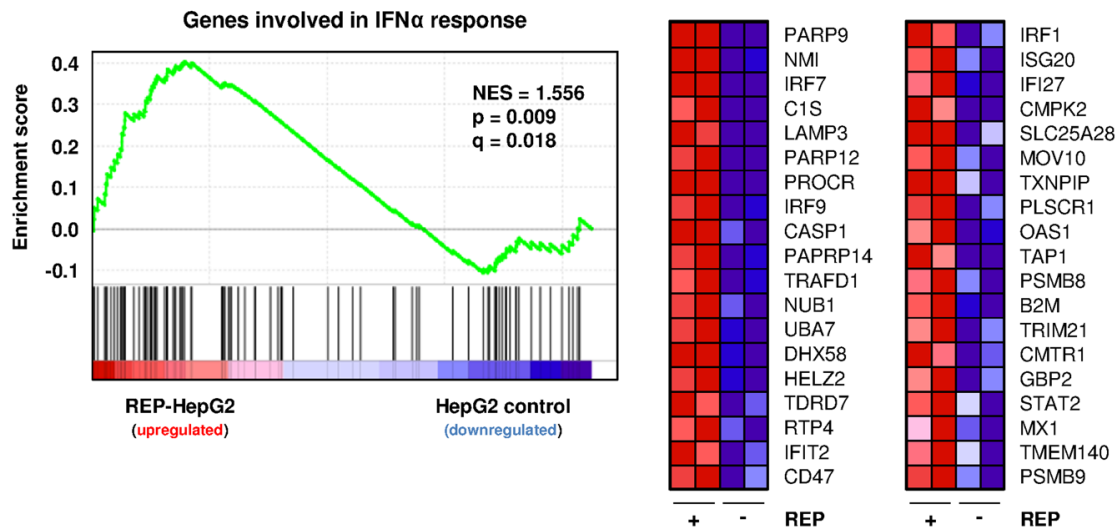

Fig. 1 Gene set enrichment analysis (GSEA) after epigenetic reprogramming of HepG2 cells. A Relevant gene sets enriched in reprogrammed HepG2 (REP-HepG2) cells. Epigenetic reprogramming of HepG2 cells was achieved with a 2.5 HM 5-AZA regimen for 10 days. Untreated HepG2 cells were used as controls. The ranking of the depicted enriched gene sets was as follows: (\#1) bile acid metabolism (molecular signature with the highest enrichment score in the HALLMARK gene datasets), (\#3) xenobiotic metabolism, (\#5) IFNa response, (\#9) IFNy response, and (\#10) complement system. The enrichment of the hepatocyte metabolism signatures was consistent with the promotion of hepatic differentiation in the REP-HepG2 cells. The GSEA was performed using microarray data from the GSE160648 data set. B Enrichment plot and heat map for the genes involved in the IFNa response in the reprogrammed versus non-reprogrammed HepG2 cells (normalized enrichment score [NES] $=1.556 ; p=0.009$ and $q=0.018$ ). See S. Table 1 for a complete list of the HALLMARK_interferon alpha genes. $1 \mathrm{mM} 5$-AZA $=244.2 \mathrm{ng} / \mathrm{mL}$.

Epigenetic reprogramming enhances the IFNa-mediated induction of ISGs

We assumed that HBV DNA inhibition after IFN $\alpha$ treatment in REP-HepG2-NTCP cells was a consequence of the re-expression of key ISGs in response to the demethylating treatment. To test this hypothesis, we evaluated the effect of IFN $\alpha$ on ISG expression in REPHepG2-NTCP cells (Fig. 3A), with a special emphasis on critical ISGs previously reported to be associated with the IFN $\alpha$-mediated antiviral response in patients ${ }^{19}$. Consistent with the GSEA data from the parental HepG2 cells (Fig. 1B), 2'-5'-oligoadenylate synthetase 1 (OAS1) and interferon-stimulated exonuclease gene 20 (ISG20) showed a significant augmentation in their expression, with level increases of $15.49 \pm 2.20$-fold and $12.32 \pm 0.47$ fold in REP-HepG2-NTCP cells compared with those in the non-reprogrammed cells after only $4 \mathrm{~h}$ of $100 \mathrm{IU} / \mathrm{mL}$ IFN $\alpha$ treatment ( $p=0.0076$ and $p<0.0001$, respectively, $t$ test) (Fig. 3B). Interestingly, the expression level of interferon regulatory factor 9 (IRF9), a transcription factor that controls the expression of numerous ISGs, was also increased by approximately threefold under these conditions. Finally, our data showed that the expression levels of apolipoprotein B mRNA-editing enzyme catalytic subunits $3 \mathrm{~F}$ and $3 \mathrm{G}$ (APOBEC $3 F$ and $A P O B E C 3 G$ ), two essential effectors of the anti-HBV response mediated by IFN $\alpha$, were also markedly increased in the REP-HepG2NTCP cells.

\section{IRF9 and OAS1 epigenetic silencing is abolished in REP- HepG2-NTCP cells}

To clarify the molecular mechanism by which ISG induction was restored in epigenetically reprogrammed cells, we first analyzed the basal expression of several genes related to the antiviral response and specific genes that characterized the IFN $\alpha / J A K / S T A T$ pathway. Our data showed that 5-AZA treatment led to a global augmentation in the expression of these markers without 
A

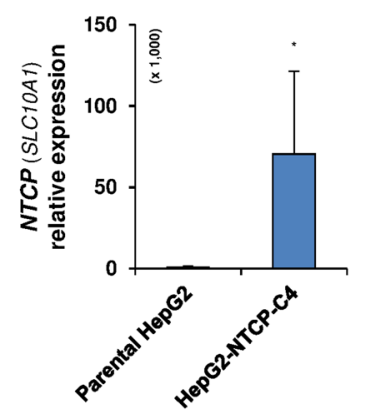

B

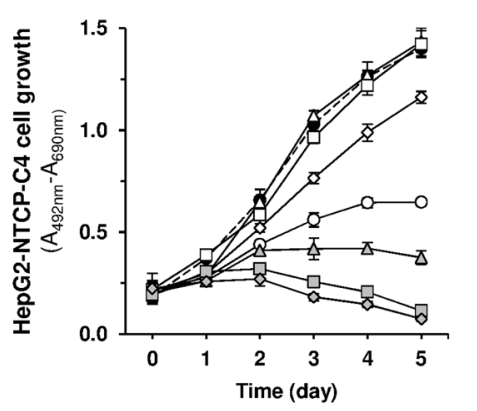

C

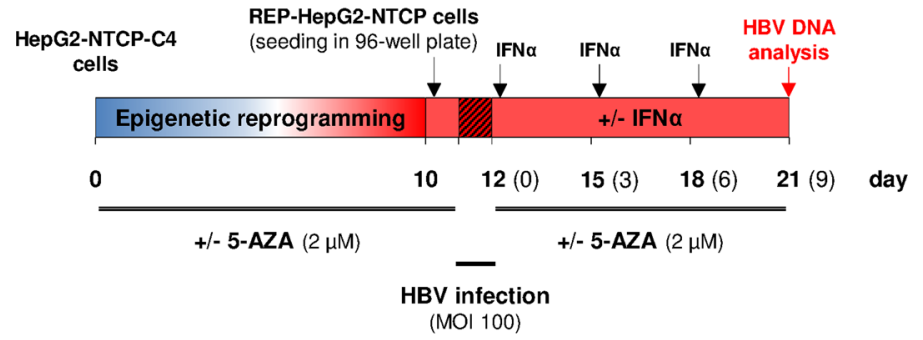

D

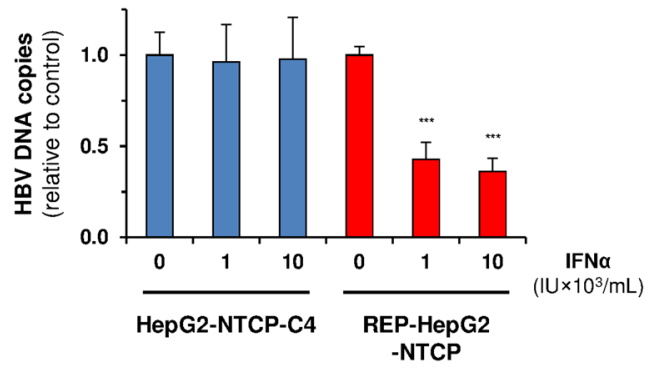

Fig. 2 Epigenetic reprogramming of HepG2-NTCP-C4 cells and evaluation of IFNa antiviral activity after HBV infection. A Relative expression level of NTCP (SLC1OA1 gene) in HepG2 and HepG2-NTCP-C4 cells. NTCP was analyzed in samples from HepG2-NTCP-C4 cell cultures at different timepoint $(n=6)$. Parental HepG2 cells were used as controls $(n=4)$. The histograms show the means \pm SD. ${ }^{*} p<0.05$ ( $t$ test). B Evaluation of 5-AZA cytotoxic effect on HepG2-NTCP-C4 cells. Twenty-four hours after seeding, the cells were treated with 5-AZA at the indicated concentrations for 5 days. HepG2-NTCP-C 4 cell viability was determined at the indicated times using an XTT assay. The 5-AZA IC50 was $4.33 \mu \mathrm{M}$ after 5 days of treatment. The data represent the means \pm standard deviation (SD). C Epigenetic reprogramming experimental design. Cells were first treated with $2 \mu \mathrm{M}$ 5-AZA for 10 days (reprogramming procedure). Twenty-four hours after seeding in 96-well plates, the reprogrammed HepG2-NTCP-C4 (REPHepG2-NTCP) cells were infected with HBV at an MOI of 100 for $24 \mathrm{~h}$. Next, REP-HepG2-NTCP cells were treated with 1,000 and 10,000 IU/mL IFNa every 3 days. Total DNA was collected for analysis after 9 days of treatment. The 5-AZA-supplemented medium was replaced daily during reprogramming and every 3 days after HBV infection. The numbers in brackets indicate the time after infection. No cytotoxic effect of IFNa was observed in the cells after treatment (S. Figure 1). D Measurement of HBV DNA levels in the HBV-infected cells after IFNa treatment. HBV DNA copies in the REP-HepG2-NTCP cells were determined by real-time quantitative PCR from $50 \mathrm{ng}$ of total DNA. Histograms represent the means \pm SD. Statistically significant differences in the HBV DNA levels related to untreated cells were achieved at ${ }^{* * *} p<0.001$ ( $t$ test).

IFN $\alpha$ stimulation (Fig. 4A). $\mathrm{z}$ scores indicated that IRF9 and $O A S 1$ were the most prominently amplified genes.

To determine whether the increased expression of IRF9 and OAS1 was the consequence of an epigenetic mechanism directly linked to the demethylating treatment during the reprogramming procedure, we analyzed the methylation profile of the genomic region upstream of the IRF9 and OAS1 transcription start site (TSS). A combined bisulfite restriction analysis (COBRA $)^{20}$ showed that specific CpG sites associated with OAS1 were differentially methylated between naive and reprogrammed HepG2-NTCP-C4 cells (Fig. 4B), delineating a differentially methylated region (DMR). The high degree of OAS1-DMR methylation measured before 5-AZA treatment (61\% versus $29 \%, p=0.0286$, Mann-Whitney $U$ test) was consistent with the epigenetic silencing of OAS1 in the non-reprogrammed HepG2-NTCP-C4 cells. Another DMR was identified upstream of the IRF9 TSS, which was also hypermethylated in HepG2-NTCP-C4 cells (Fig. 4C). Similar to its effect on OAS1-DMR, 5-AZA 


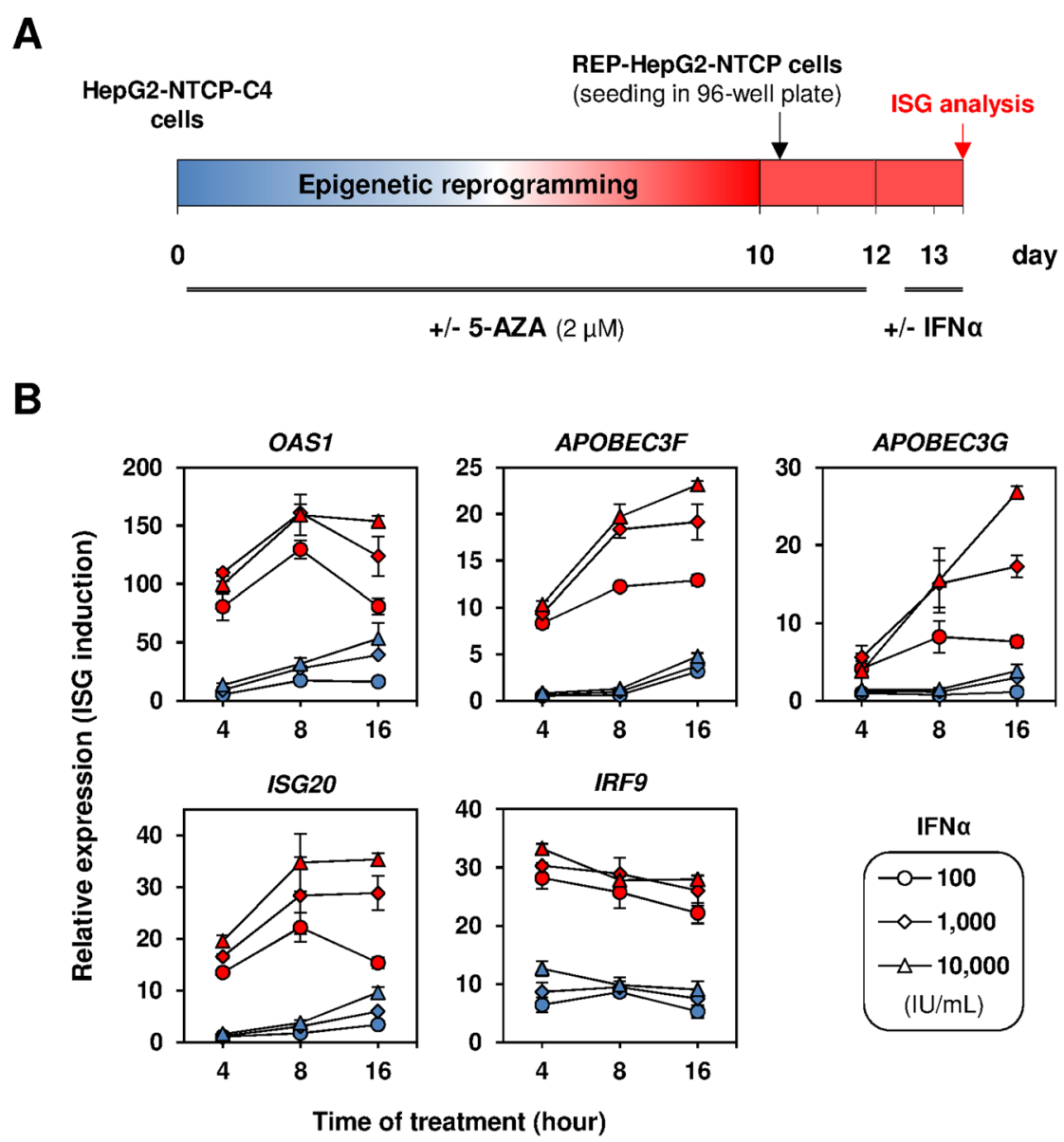

Fig. 3 ISG expression analysis in IFNa-stimulated REP-HepG2-NTCP cells. A Experimental design. After epigenetic reprogramming using $2 \mu$ M 5AZA for 10 days, REP-HepG2-NTCP cells were treated with different concentrations of IFNa for 4, 8, and $16 \mathrm{~h}$. B Relative expression levels of 2'-5'oligoadenylate synthetase 1 (OAS1), interferon-stimulated exonuclease gene 20 (ISG20), apolipoprotein B mRNA-editing enzyme catalytic subunits $3 \mathrm{~F}$ and $3 G(A P O B E C 3 F$ and $A P O B E C 3 G)$, and interferon regulatory factor 9 (IRF9). Total RNA was extracted at the indicated times, and the relative gene expression levels were determined by real-time quantitative PCR. The data obtained from REP-HepG2-NTCP cells and naive HepG2-NTCP-C4 cells are depicted in red and blue, respectively, and show the means \pm SD.

treatment promoted major demethylation of IRF9-DMR (23\% versus $100 \%, p=0.0022$, Mann-Whitney $U$ test), which was associated with the epigenetic unmasking of this gene. The methylation profiles of other critical ISGs, such as ISG20 and APOBEC3G, were also determined by COBRA. However, the COBRA data showed no methylation in the CpG sites upstream of the ISG2O or APO$B E C 3 G$ TSS (S. Figure 2), suggesting that a mechanism independent of DNA methylation was responsible for the increased expression of these two genes in REP-HepG2NTCP cells.

\section{Epigenetic reprogramming increases NTCP expression in HepG2-NTCP-C4 cells}

HepG2-NTCP cell lines are characterized by stable overexpression of NTCP, which confers the susceptibility of these cells to HBV infection ${ }^{12,13}$. In line with our previous studies using the parental HepG2 cells $^{17}$, we observed that NTCP expression was increased in REPHepG2-NTCP cells compared with control HepG2NTCP-C4 cells (Fig. 5A). Although no DMR was found in the NTCP promoter, two distinct CpG sites appeared differentially methylated in the control and reprogrammed cells (Fig. 5B). These CpG sites were located $948 \mathrm{bp}$ upstream and $172 \mathrm{bp}$ downstream of the NTCP TSS and exhibited methylation of $44 \%$ and $71 \%$, respectively, in naive HepG2-NTCP-C4 cells, as determined by COBRA. The augmentation in NTCP levels was correlated with significant demethylation of these two $\mathrm{CpG}$ sites in REP-HepG2-NTCP cells, with methylation of $24 \%$ for CpG site \#1 and 42\% for CpG site \#6 ( $p=0.0003$ and $p=0.0018$, respectively, $t$ test).

\section{CDM-3008 shows antiviral effects in REP-HepG2-NTCP cells}

To test the potential of epigenetic reprogramming for the evaluation of experimental IFN-like compounds, REP- 
A
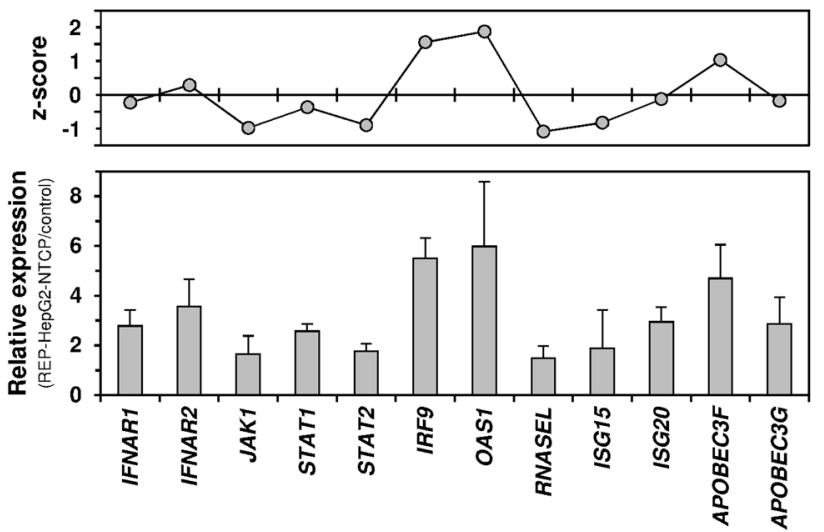

B
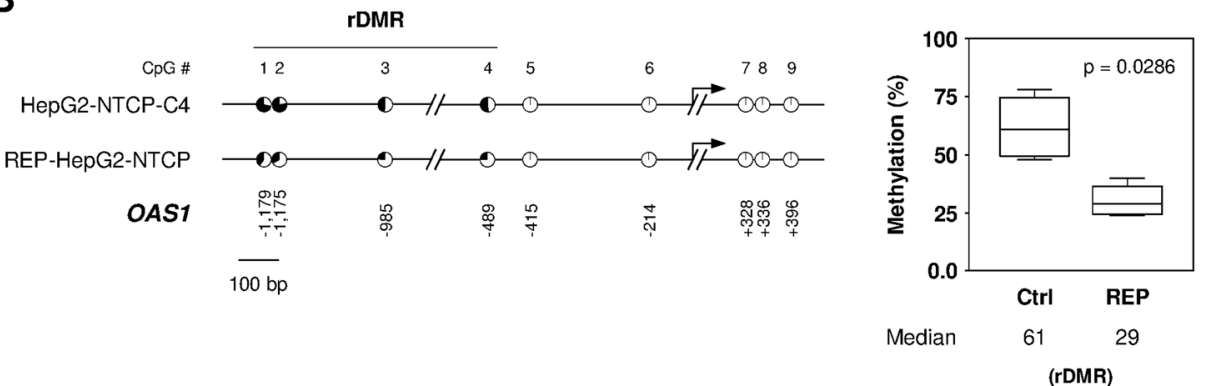

C
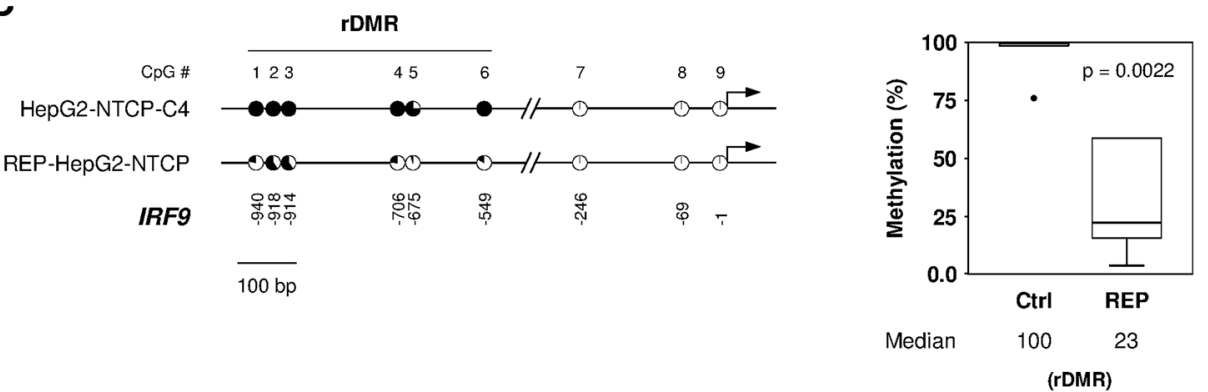

Fig. 4 Analysis of OAS1 and IRF9 methylation after epigenetic reprogramming. A Relative expression levels of interferon-alpha and interferonbeta receptor subunit 1 and 2 (IFNAR1 and IFNAR2), Janus kinase 1 (JAK1), signal transducer and activator of transcription 1 and 2 (STAT1 and STAT2), ribonuclease L (RNASEL), ISG15 ubiquitin-like modifier (ISG15), ISG20, APOBEC3F, and APOBEC3G. The data show gene expression fold changes in the REP-HepG2-NTCP cells relative to non-reprogrammed control cells and $z$ scores for each gene relative to all analyzed genes $(N=12)$. B Comparison of OAS1 and C IRF9 methylation profiles between REP-HepG2-NTCP cells and HepG2-NTCP-C4 cells. The COBRA data showed reprogramming DMRs (rDMRs) located from 549 to $940 \mathrm{bp}$ and from 489 to $1179 \mathrm{bp}$ upstream of the OAS1 and IRF9 TSSs, respectively. The black arrows indicate the OAS1 and IRF9 TSSs and the black circles represent the methylation percentage for each analyzed CpG site. The boxplots show the differential methylation levels of OAS1-rDMR and IRF9-rDMR in the REP-HepG2-NTCP cells (REP) compared with those of the control HepG2-NTCP-C4 cells (Ctrl). Significant differences in methylation levels were achieved at ${ }^{* *} p<0.01$ for OAS1-rDMR and ${ }^{*} p<0.05$ for IRF9-rDMR (Mann-Whitney $U$ test).

HepG2-NTCP cells were exposed to CDM-3008, an IFN agonist previously characterized by high-throughput chemical library screening ${ }^{21,22}$. Our results showed that HBV DNA levels decreased by $44.58 \% \pm 0.09 \%(p=$ $0.0002, t$ test $)$ and $39.02 \% \pm 0.07 \%(p=0.0115, t$ test $)$ in the infected REP-HepG2-NTCP cells when treated with 1 and $10 \mu \mathrm{M}$ CDM-3008, respectively (Fig. 6A). Similar to IFN $\alpha$, HBV DNA levels remained unchanged after CDM3008 treatment in naive HepG2-NTCP-C4 cells. HBV
DNA inhibition after CDM-3008 treatment was associated with the marked induction of ISGs in the REPHepG2-NTCP cells, which was limited in nonreprogrammed cells (Fig. 6B). We confirmed that no cytotoxic effect was induced in cells after 5-AZA and CDM-3008 treatment (S. Figure 1B). Considering that CDM-3008 antiviral action is closely correlated to the levels of expression of cell-surface interferon-alpha and -beta receptor subunit $2(\text { IFNAR2 })^{21,23}$, we analyzed ISG 
A

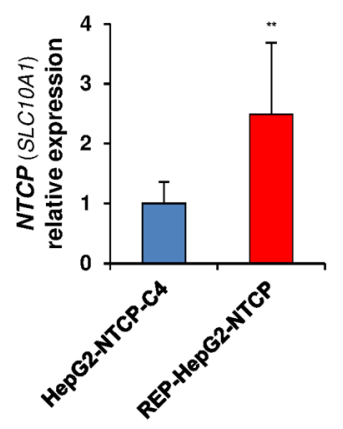

B
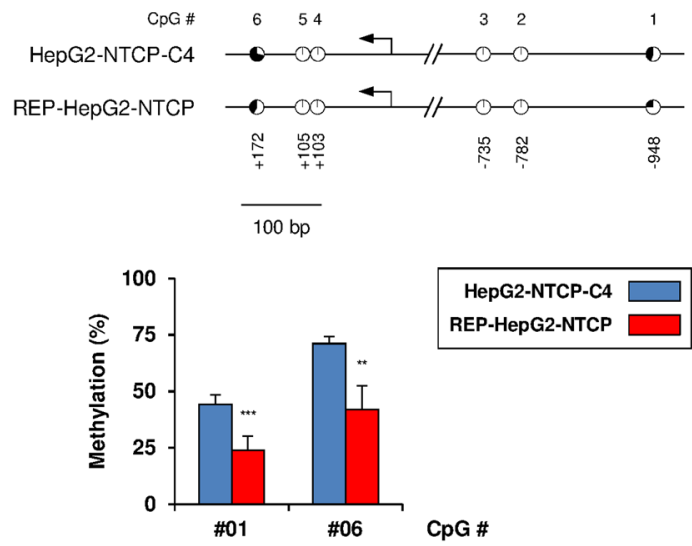

Fig. 5 Expression and methylation levels of NTCP in REP-HepG2-NTCP cells. A Relative expression level of NTCP (SLC10A1 gene) in REP-HepG2NTCP cells. Non-reprogrammed HepG2-NTCP-C4 cells were used as controls. The histograms show the means \pm SD. ${ }^{* *} p<0.01$ ( $t$ test). B Methylation status of the NTCP promoter region as determined by COBRA. Two CpG sites located 948 bp upstream and 172 bp downstream of the NTCP TSS were found to be differentially methylated between the REP-HepG2-NTCP cells and control HepG2-NTCP-C4 cells. A significant difference in CpG methylation levels was reached at $p<0.001$ ( $C p G \# 01)$ and $p<0.01$ (CpG \#06) ( $t$ test).

induction by CDM-3008 after knocking down IFNAR2 using siRNAs. First, the efficiency of the siRNAs to inhibit IFNAR2 specifically was validated (S. Figure 3). Next, our data showed that IFNAR2 silencing jeopardized the induction of ISGs after CDM-3008 treatment in REPHepG2-NTCP cells (Fig. 6C). For instance, OAS1 expression was decreased by $\sim 63 \%$ in the CDM-3008stimulated REP-HepG2-NTCP cells after IFNAR2 knockdown ( $p=0.0003, t$ test). Combined, our data supported the adequacy of the REP-HepG2-NTCP cell system for use in the functional assessment of IFN-like compounds, such as CDM-3008 (Fig. 6D).

\section{Discussion}

To establish an ideal cell culture-based system for HBV studies, consistent virus infection/replication rates and antiviral responses are required. This study provides evidence for efficient epigenetic reprogramming of the HBVsusceptible HepG2-NTCP-C4 clone toward IFN $\alpha$ and CDM-3008-responsive cells. We report that restoration of the antiviral properties of these molecules was associated with the epigenetic unmasking of key ISGs through DMR demethylation in REP-HepG2-NTCP cells.

Epigenetic processes are essential for orchestrating gene expression in eukaryotic organisms ${ }^{24,25}$. Cancer cells are usually characterized by aberrant hypermethylation patterns restricted to $\mathrm{CpG}$-rich regions that lead to the silencing of critical genes and microRNAs associated with tumor suppression or cell differentiation ${ }^{26-29}$. Given the reversible nature of epigenetic modifications ${ }^{30,31}$, the use of demethylating drugs is promising for cell epigenetic reprogramming, as these compounds are unique tools for reversing the cancer phenotype and restoring tissuespecific gene expression. We previously reported the efficient reactivation of epigenetically silenced genes associated with cell differentiation through the use of a demethylating treatment in hepatic and pancreatic cancer cells ${ }^{16-18}$. In the present work, we connected the absence of an antiviral response in naive HepG2-NTCP-C4 cells with the epigenetic silencing of two major ISGs, OAS1 and IRF9. In contrast, epigenetic reprogramming efficiently restored the IFN-stimulated induction of OAS1 and IRF9. Recovery of antiviral activity in REP-HepG2NTCP cells was also associated with the induction of additional ISGs, such as ISG2O and APOBEC3G, independent of a DMR-mediated regulation mechanism. Our findings suggest that ISG2O and APOBEC3G probably belong to a set of ISGs, which are not directly reactivated by the demethylating compound during epigenetic reprogramming. Thus, HepG2-NTCP-C4 cell reprogramming is most likely the consequence of a sequential reactivation of ISGs, characterized first (i) by the direct demethylation and re-expression of specific epigenetically controlled ISGs (e.g., OAS1) and key transcription factors, such as IRF9 and (ii) by the subsequent initiation of the global transcription of ISGs (Fig. 6D).

As the main target of HBV infection ${ }^{32}$, human hepatocytes appear to be the most appropriate model for antiHBV drug screening. However, access to PHHs is limited because of the scarcity of liver donors and the substantial price of this biological material. Furthermore, the inability to expand PHHs in vitro and the extreme variability of the antiviral response among donors limit their utilization. The HepaRG cell model is an interesting substitute for 
A

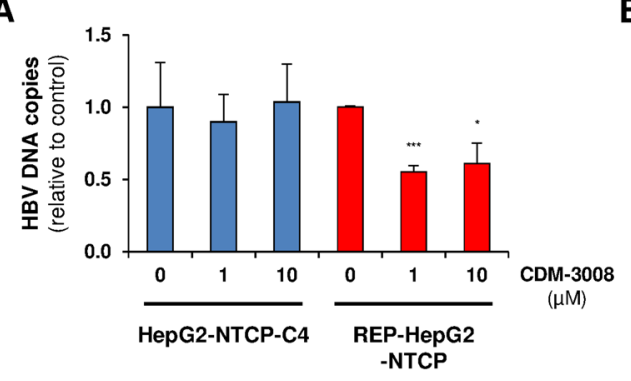

C

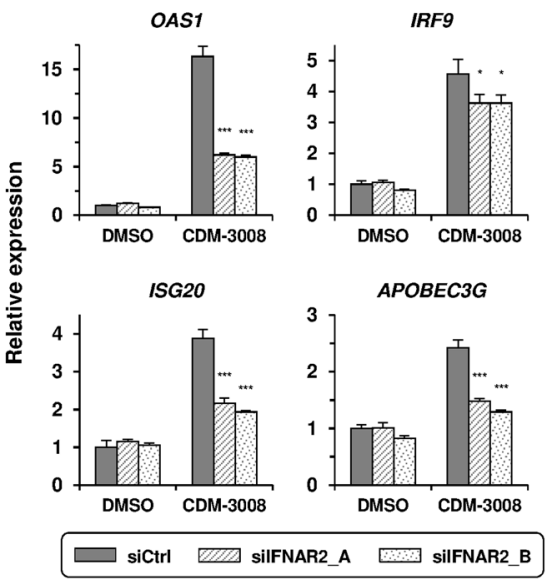

B

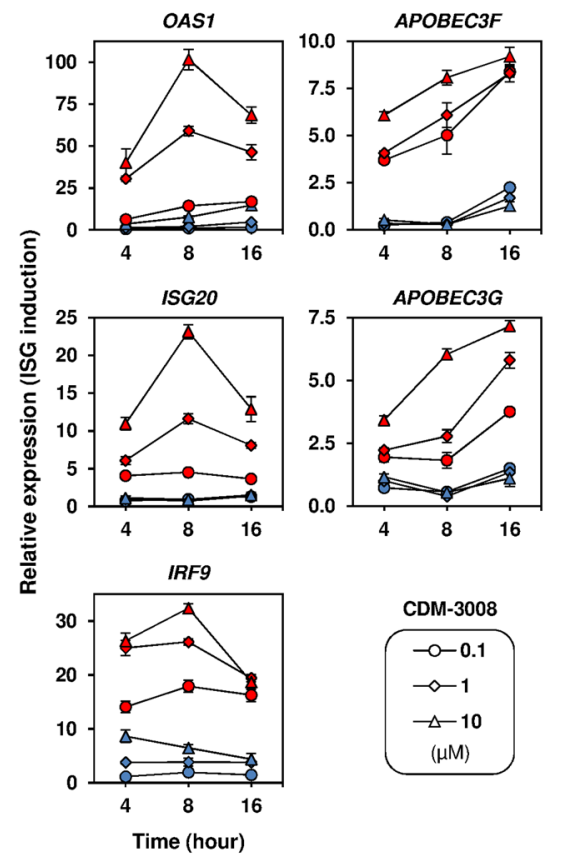

D

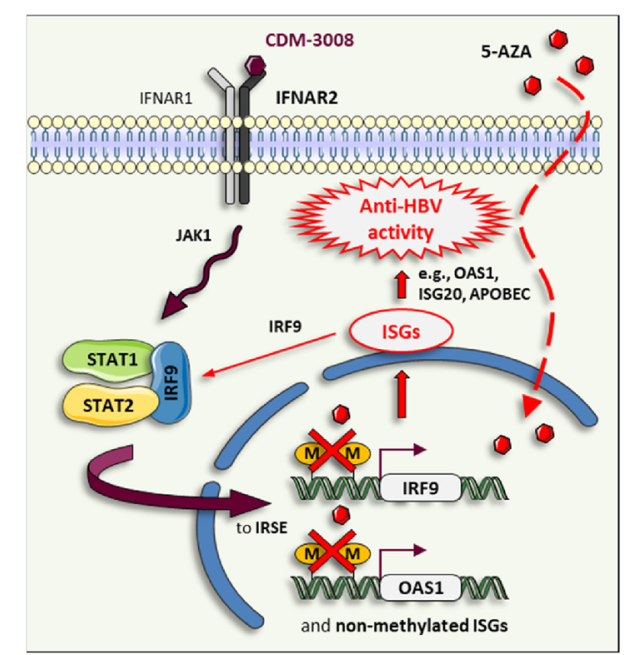

Fig. 6 Assessment of CDM-3008 activity in REP-HepG2-NTCP cells. A Measurement of HBV DNA levels in HBV-infected cells after CDM-3008 treatment. Following epigenetic reprogramming ( $2 \mu \mathrm{M}$ 5-AZA for 10 days) and HBV infection (MOI of 100 for $24 \mathrm{~h}$ ), REP-HepG2-NTCP cells were treated with 1 and $10 \mu \mathrm{M}$ CDM-3008 every 3 days. Total DNA was collected for analysis after 9 days of treatment. No cytotoxic effect was induced by the compound in cells after treatment (S. Figure 1). Statistically significant differences in HBV DNA levels relative to the level in the untreated cells were achieved at ${ }^{* *} p<0.001$ and ${ }^{*} p<0.05$ with 1 and $10 \mu \mathrm{M}$ CDM-3008, respectively ( $t$ test). B Relative expression levels of OAS1, ISG20, APOBEC3F, APOBEC3G, and IRF9 after CDM-3008 treatment at the indicated times and concentrations. The data from the REP-HepG2-NTCP and naive HepG2NTCP-C4 cells are depicted in red and blue, respectively. C Relative expression of OAS1, ISG20, APOBEC3G, and IRF9 after IFNAR2 knockdown. Fortyeight hours after transfection with control siRNAs (siCtrl) or IFNAR2 siRNAs (silFNAR2_A and silFNAR2_B), REP-HepG2-NTCP cells were treated with $1 \mu \mathrm{M}$ CDM-3008 for $8 \mathrm{~h}$. Significance related to siCtrl-transfected cells was evaluated by $t$ test: ${ }^{*} p<0.05$ and ${ }^{* * *} p<0.001$. All histograms depicted in the figure represent the means \pm SD. D Proposed mechanism by which epigenetic reprogramming of HepG2-NTCP-C4 cells restores, in part, via OAS1 and IRF9 epigenetic unmasking, ISG induction and anti-HBV activity in response to CDM-3008 treatment. 
PHHs for use in HBV studies ${ }^{9}$, but these cells require several weeks of treatment with dimethyl sulfoxide (DMSO) to fully differentiate into a mixture of bile epithelial cells and hepatocytes ${ }^{33}$, which can be a source of bias for reliable antiviral response studies. A significant step forward has been made since the discovery of the HBV entry receptor NTCP and the development of various NTCP-overexpressing $\mathrm{HCC}$ cell models, such as HepG2-NTCP cells ${ }^{12-14}$. The HepG2-NTCP cell lines probably represent the most common and popular models for HBV studies because of the ease of access of these cells, their high transfection rate, and their consistent antiviral response inherent to their clonogenic nature. In an elegant study, Qiao and collaborators established various HepG2-NTCP clones that stably overexpressed $N T C P$ at different levels and demonstrated that HBV permissiveness was tightly correlated with NTCP protein levels ${ }^{34}$. The authors also showed that HBV replication was significantly promoted by treating the HBV-infected HepG2-NTCP clones with DMSO and hydrocortisone. It would be interesting to apply the epigenetic reprogramming protocol to improve the antiviral activity in this optimized HBV cell culture model for potential drugscreening applications.

In conclusion, our study demonstrates that epigenetic reprogramming of HepG2-NTCP-C4 cells using 5-AZA promotes the anti-HBV action of IFNa. REP-HepG2NTCP cells also appear appropriate for the functional and therapeutic assessment of IFN-like compounds. Additional investigation will be essential to determine the impact of epigenetic modifications on HBV genome and the value of epigenetically reprogrammed cells for future drug-screening applications. At last, it is certainly appealing to consider that epigenetic reprogramming could also be valuable for improving other cell-virus culture systems that lack appropriate cell-mediated antiviral responses for treatment evaluation.

\section{Materials and methods}

\section{Human hepatic cell lines and reagents}

HepG2-NTCP-C4 cells and HepG2.2.15.7 cells were obtained from the National Institute of Infectious Diseases (Tokyo, Japan). The HepG2-NTCP-C4 cells were maintained in a Dulbecco's modified Eagle's medium (DMEM)/F-12, GlutaMAX mixture (Gibco) supplemented with insulin $(5 \mu \mathrm{g} / \mathrm{mL}$; Wako), hydrocortisone $(50 \mu \mathrm{M}$; Wako), $10 \mathrm{mM}$ HEPES (Gibco), penicillin (100 IU/mL; Gibco), streptomycin $(100 \mu \mathrm{g} / \mathrm{mL} ;$ Gibco), and $10 \%$ fetal bovine serum (FBS; Gibco). The HepG2.2.15.7 cells were maintained in a DMEM/F-12, GlutaMAX mixture supplemented with insulin $(5 \mu \mathrm{g} / \mathrm{mL}$; Wako), penicillin (100 IU/mL), streptomycin $(100 \mu \mathrm{g} /$ $\mathrm{mL}$ ), and 10\% FBS. HepG2 cells were purchased from the American Type Culture Collection and maintained in
DMEM (Gibco) supplemented with $2 \mathrm{mM}$ L-glutamine (Gibco), penicillin $(50 \mathrm{IU} / \mathrm{mL})$, streptomycin $(50 \mu \mathrm{g} / \mathrm{mL})$, and $10 \%$ FBS. The demethylating compound 5-AZA (PubChem CID: 9444) was purchased from Sigma. 5-AZA was dissolved in phosphate-buffered saline (PBS) as a $10 \mathrm{mM}$ stock solution, filtered $(0.22 \mu \mathrm{M})$, and stored at $-20^{\circ} \mathrm{C}$ in aliquots. The 5-AZA-supplemented medium was prepared every day. IFN $\alpha$ solution was obtained from Sumitomo Dainippon Pharma. CDM-3008 (RO8191; PubChem CID: 2768133) was obtained from SIGMA and dissolved in DMSO as a $1,000 \mu \mathrm{M}$ working solution before use.

\section{HBV production and cell infection}

HBV particles were collected from HepG2.2.15.7 cells, which stably express and replicate the virus (genotype D). The culture medium containing viral particles was collected from confluent cells. After filtration $(0.22 \mu \mathrm{M})$, the medium was incubated with a mixture of $23 \% \mathrm{NaCl}$ and $50 \%$ PEG 8000 for at least $2 \mathrm{~h}$ at $4^{\circ} \mathrm{C}$. Next, HBV particles were purified by ultracentrifugation, washed with PBS, resuspended in cell culture medium, and stored at $-80^{\circ} \mathrm{C}$ until used for cell infection. REP-HepG2-NTCP cells and HepG2-NTCP-C4 cells were seeded in 96-well plates $24 \mathrm{~h}$ before infection. The cells were infected using a mix of $10 \mu \mathrm{L} 40 \%$ PEG 8000 and $90 \mu \mathrm{L}$ cell culture medium (without 5-AZA) containing HBV particles at an MOI of 100 for $24 \mathrm{~h}$.

\section{Cell transfection}

REP-HepG2-NTCP cells were seeded at a density of 200,000 cells $/ \mathrm{cm}^{2}$ in $35-\mathrm{mm}$-diameter culture dishes and transfected the next day using TransFectin lipid reagent (Bio-Rad). Cells were incubated with a transfection mix containing $100 \mathrm{nmol} / \mathrm{L}$ siRNA and $5 \mu \mathrm{L}$ of TransFectin in a $1.2 \mathrm{~mL}$ total volume of serum- and antibiotic-free OptiMEM (Invitrogen) for $5 \mathrm{~h}$. Human IFNAR2 siRNAs (IDs s7185 and s7186 for siIFNAR2_A and siIFNAR2_B, respectively) (Ref. \#4390824) and Silencer Select Negative Control \#1 siRNA (siCtrl; Ref. \#4390843) were purchased from Ambion.

\section{Total DNA and RNA isolation}

Total DNA was purified from REP-HepG2-NTCP cells and naive HepG2-NTCP-C4 cells using an Agencourt DNAdvance Genomic DNA Isolation kit and a Biomek NXp automatic dispenser from Beckman Coulter according to the manufacturer's instructions. Total RNA was purified using an Agencourt RNAdvance Cell v2 and a Biomek NXp automatic from Beckman Coulter according to the manufacturer's instructions. Before elution, the RNA samples were treated with five units DNase I (Nippon Gene) at $25^{\circ} \mathrm{C}$ for $15 \mathrm{~min}$. For the microarray analysis, total RNA was purified from HepG2 cells using a 
miRNeasy Mini kit (Qiagen) according to the manufacturer's recommendations, and samples were treated with $1 \mathrm{~mL}$ of DNase at $37^{\circ} \mathrm{C}$ for $30 \mathrm{~min}$ using a TURBO DNA-free kit (Ambion). DNA and RNA sample concentrations were determined using a DeNovix spectrophotometer.

\section{Gene expression microarray and GSEA}

After purification, RNA samples from 5-AZA-treated HepG2 cells ( $2.5 \mu \mathrm{M}$ for 10 days) were subjected to a gene expression microarray. Untreated HepG2 cells were used as controls. RNA labeling and hybridization were performed using an Agilent one-color low RNA input linear amplification kit labeling protocol and an Agilent onecolor gene expression hyb/wash protocol (Agilent Technologies). The microarray slides were scanned in an Agilent G2505C Microarray Scanner at 3-micron resolution (Agilent Technologies). The raw data were processed using Agilent's Feature Extraction Software version 10.7.3.1 to analyze the array and calculate the intensities of the measured spots. The Agilent Gene Expression Platform was Agilent-039494. GSEA was performed using the HALLMARK gene datasets (http://software. broadinstitute.org/gsea).

\section{mRNA and HBV DNA real-time quantitative PCR}

To evaluate gene expression levels, cDNAs were first synthesized from $1 \mu \mathrm{g}$ of purified RNA using PrimeScript RT Master Mix (TaKaRa) according to the manufacturer's recommendations. Real-time quantitative PCR was performed using TB Green Premix Ex Taq II (TaKaRa) and a CFX96 Real-Time PCR Detection System (Bio-Rad). After initial denaturation at $95^{\circ} \mathrm{C}$ for $30 \mathrm{sec}$, the thermal cycles were repeated 40 times as follows: $95^{\circ} \mathrm{C}$ for $5 \mathrm{sec}$ and $60^{\circ} \mathrm{C}$ for $30 \mathrm{sec}$. The housekeeping gene glyceraldehyde 3phosphatase dehydrogenase (GAPDH) was used to normalize the cDNA levels. The sequences of the human primers used for gene amplification are shown in $\mathbf{S}$. Table 2. HBV DNA levels were determined by real-time quantitative PCR using Probe qPCR mix (TaKaRa) from 50 ng of total DNA. The PCR conditions were $95^{\circ} \mathrm{C}$ for $1 \mathrm{~min}$, followed by 50 cycles of $95^{\circ} \mathrm{C}$ for $10 \mathrm{sec}$ and $60^{\circ} \mathrm{C}$ for $30 \mathrm{sec}$. The HBV DNA TaqMan probe sequence was 5'-[FAM] TATCGCT GGATGTGTCTGCGGCGT[TAM]-3'. The sequences of the specific primers used for HBV DNA detection were $5^{\prime}$ ACTCACCAACCTCCTGTCCT-3' (forward primer) and 5'-GACAAACGGGCAACATACCT-3' (reverse primer).

\section{DNA methylation analysis}

COBRA was used to assess the methylation status of OAS1, IRF9, ISG20, APOBEC3G, and NTCP before and after epigenetic reprogramming of HepG2-NTCP-C4 cells. An in silico analysis using the UCSC Genome Bioinformatics tool (http://genome.ucsc.edu) was performed to identify the $\mathrm{CpG}$ sites located upstream of the TSS of these genes. MethPrimer (http://www.urogene. org/methprimer) was used to design the COBRA primers required to amplify the genomic regions containing the CpGs of interest (S. Table 3). One microgram of genomic DNA was subjected to bisulfite conversion treatment using an EpiTect Plus kit (QIAGEN). Next, COBRA PCR was performed as follows: after an initial denaturation step of $94^{\circ} \mathrm{C}$ for $3 \mathrm{~min}$, the following thermal cycles were repeated 40 times: $94^{\circ} \mathrm{C}$ for $10 \mathrm{sec}, 55^{\circ} \mathrm{C}$ for $50 \mathrm{sec}$, and $72^{\circ} \mathrm{C}$ for $1 \mathrm{~min}$. Each COBRA PCR was performed in a total volume of $10 \mu \mathrm{L}$, which contained 0.5 units of Hot Start Taq polymerase (TaKaRa), 10 pmol of primers, and $1 \mu \mathrm{L}$ of bisulfite-treated DNA. After PCR amplification, COBRA PCR products were digested with three units of restriction enzyme. Finally, the restriction products were separated by polyacrylamide gel electrophoresis and visualized by ethidium bromide staining. The bands were densitometrically analyzed using ImageJ software (v1.50i, National Institutes of Health, USA) to quantify the unmethylated $(U)$ and methylated $(M)$ restriction fragments. The methylation levels were calculated for each locus using the formula $(M \times 100) /(M+U)$; they are expressed as methylation percentages.

\section{Cell viability assay}

For the evaluation of the time- and dose-dependent cytotoxicity of 5-AZA, HepG2-NTCP-C4 cells were seeded at 15,000 cells/well in 96-well plates and treated with 5AZA at the indicated concentrations. The 5-AZAsupplemented medium was changed every 2 days. Cell viability was determined at the indicated times using a Cell Proliferation Kit II from Roche, according to the manufacturer's instructions (XTT assay). Absorbance at $492 \mathrm{~nm}$ and $690 \mathrm{~nm}$ was measured using an EnSight Multimode Plate Reader (PerkinElmer). REP-HepG2-NTCP cell and HepG2-NTCP-C4 cell viabilities were determined after IFN $\alpha$ or CDM-3008 treatment using the same method.

\section{Statistical analysis}

All experimental data are presented as the means \pm SD. Student's $t$ test was performed to estimate statistical significance. The equality of the variances was tested using an $F$ test, and a correction was performed in cases of unequal variance (Welch $t$ test). All $p$ values were twotailed. The statistical significance of differences in OAS1DMR and IRF9-DMR methylation levels between reprogrammed and control cells was assessed using a Mann-Whitney $U$ test. All depicted data are representative of at least three independent experiments.

\footnotetext{
Acknowledgements

We thank Ting Su, Mariko Toguchi, Shoko Higuchi, Kaori Yanaka, and Noriko Tanaka for their assistance. The authors are also grateful to Izuho Hatada for
} constructive comments on the study. This work was supported by a grant from 
the Japan Agency for Medical Research and Development (J JP21fk0310112), a Grant-in-Aid for Scientific Research (20K07604) from the Japan Society for the Promotion of Science, and Riken Incentive Research Projects 2019.

\section{Author details}

${ }^{1}$ Liver Cancer Prevention Research Unit, RIKEN Cluster for Pioneering Research, Wako, Japan. ${ }^{2}$ Department of Translational Research, Joint Research Center for Human Retrovirus Infection, Kagoshima University, Kagoshima, Japan. ${ }^{3}$ Research Center for Drug and Vaccine Development, National Institute of Infectious Diseases, Tokyo 162-8640, Japan. ${ }^{4}$ Department of Virology II, National Institute of Infectious Diseases, Tokyo, Japan. ${ }^{5}$ Department of Molecular and Cellular Medicine, Tokyo Medical University, Tokyo, Japan. ${ }^{6}$ Department of Laboratory Medicine, The Jikei University School of Medicine, Tokyo, Japan

\section{Data availability}

Microarray data are deposited in the NCBI Gene Expression Omnibus (GEO) under the accession number GSE160648.

\section{Ethics statement}

All experiments have been performed in accordance with the regulations of the ethics committee of RIKEN.

\section{Conflict of interest}

The authors declare no competing interests.

\section{Publisher's note}

Springer Nature remains neutral with regard to jurisdictional claims in published maps and institutional affiliations.

Supplementary information The online version contains supplementary material available at https://doi.org/10.1038/s41420-021-00515-y.

Received: 30 March 2021 Revised: 14 April 2021 Accepted: 1 May 2021 Published online: 02 June 2021

\section{References}

1. Schweitzer, A., Horn, J., Mikolajczyk, R. T., Krause, G. \& Ott, J. J. Estimations of worldwide prevalence of chronic hepatitis B virus infection: a systematic review of data published between 1965 and 2013. Lancet 386, 1546-1555 (2015).

2. Forner, A., Reig, M. \& Bruix, J. Hepatocellular carcinoma. Lancet 391, 1301-1314 (2018).

3. Bray, F. et al. Global cancer statistics 2018: GLOBOCAN estimates of incidence and mortality worldwide for 36 cancers in 185 countries. CA Cancer J. Clin. 68, 394-424 (2018).

4. Tang, L. S. Y., Covert, E., Wilson, E. \& Kottilil, S. Chronic hepatitis B infection: a review. JAMA 319, 1802-1813 (2018).

5. Bhattacharya, D. \& Thio, C. L. Review of hepatitis B therapeutics. Clin. Infect. Dis. 51, 1201-1208 (2010)

6. Sadler, A. J. \& Williams, B. R. Interferon-inducible antiviral effectors. Nat. Rev. Immunol. 8, 559-568 (2008).

7. Liang, T. J. et al. Present and future therapies of hepatitis B: From discovery to cure. Hepatology 62, 1893-1908 (2015).

8. Sung, J. J. et al. Intrahepatic hepatitis B virus covalently closed circular DNA can be a predictor of sustained response to therapy. Gastroenterology $\mathbf{1 2 8}$, 1890-1897 (2005)

9. Gripon, P. et al. Infection of a human hepatoma cell line by hepatitis B virus. Proc. Natl Acad. Sci. USA 99, 15655-15660 (2002).
10. Belloni, L. et al. IFN-alpha inhibits HBV transcription and replication in cell culture and in humanized mice by targeting the epigenetic regulation of the nuclear cccDNA minichromosome. J. Clin. Invest. 122, 529-537 (2012).

11. Lucifora, J. et al. Specific and nonhepatotoxic degradation of nuclear hepatitis $B$ virus cccDNA. Science 343, 1221-1228 (2014).

12. Yan, $\mathrm{H}$. et al. Sodium taurocholate cotransporting polypeptide is a functional receptor for human hepatitis B and D virus. eLife 1, e00049 (2012).

13. Iwamoto, M. et al. Evaluation and identification of hepatitis B virus entry inhibitors using HepG2 cells overexpressing a membrane transporter NTCP. Biochem. Biophys. Res. Commun. 443, 808-813 (2014).

14. Michailidis, E. et al. A robust cell culture system supporting the complete life cycle of hepatitis B virus. Sci. Rep. 7, 16616 (2017).

15. Shen, F. et al. Hepatitis B virus sensitivity to interferon-alpha in hepatocytes is more associated with cellular interferon response than with viral genotype. Hepatology 67, 1237-1252 (2018).

16. Gailhouste, L. et al. Epigenetic reprogramming of human hepatoma cells: a low-cost option for drug metabolism assessment. Cell. Mol. Gastroenterol. Hepatol. 5, 454-457 (2017).

17. Gailhouste, L. et al. Differentiation therapy by epigenetic reconditioning exerts antitumor effects on liver cancer cells. Mol. Ther. 26, 1840-1854 (2018).

18. Gailhouste, L., Liew, L. C., Hatada, I., Nakagama, H. \& Ochiya, T. Epigenetic reprogramming using 5 -azacytidine promotes an anti-cancer response in pancreatic adenocarcinoma cells. Cell Death Dis. 9, 468 (2018).

19. Jansen, L. et al. An intrahepatic transcriptional signature of enhanced immune activity predicts response to peginterferon in chronic hepatitis B. Liver Int. $\mathbf{3 5}$ 1824-1832 (2015)

20. Xiong, Z. \& Laird, P. W. COBRA: a sensitive and quantitative DNA methylation assay. Nucleic Acids Res. 25, 2532-2534 (1997).

21. Konishi, $H$. et al. An orally available, small-molecule interferon inhibits viral replication. Sci. Rep. 2, 259 (2012).

22. Takahashi, N. et al. Development of an anti-hepatitis B virus (HBV) agent through the structure-activity relationship of the interferon-like small compound CDM-3008. Bioorg. Med. Chem. 27, 470-478 (2019).

23. Furutani, Y. et al. An interferon-like small chemical compound CDM-3008 suppresses hepatitis B virus through induction of interferon-stimulated genes. PlOS ONE 14, e0216139 (2019).

24. Jaenisch, R. \& Bird, A. Epigenetic regulation of gene expression: how the genome integrates intrinsic and environmental signals. Nat. Genet. 33, 245-254 (2003).

25. Li, E. Chromatin modification and epigenetic reprogramming in mammalian development. Nat. Rev. Genet. 3, 662-673 (2002).

26. Esteller, M. Cancer epigenomics: DNA methylomes and histone-modification maps. Nat. Rev. Genet. 8, 286-298 (2007).

27. Deaton, A. M. \& Bird, A. CpG islands and the regulation of transcription. Genes Dev. 25, 1010-1022 (2011).

28. Gailhouste, L. et al. miR-148a plays a pivotal role in the liver by promoting the hepatospecific phenotype and suppressing the invasiveness of transformed cells. Hepatology 58, 1153-1165 (2013).

29. Gailhouste, L. et al. MEG3-derived miR-493-5p overcomes the oncogenic feature of IGF2-miR-483 loss of imprinting in hepatic cancer cells. Cell Death Dis. 10, 553 (2019).

30. Dawson, M. A. \& Kouzarides, T. Cancer epigenetics: from mechanism to therapy. Cell 150, 12-27 (2012).

31. Dawson, M. A. The cancer epigenome: concepts, challenges, and therapeutic opportunities. Science 355, 1147-1152 (2017).

32. Protzer, U., Maini, M. K. \& Knolle, P. A. Living in the liver: hepatic infections. Nat Rev. Immunol. 12, 201-213 (2012).

33. Antherieu, S. et al. Stable expression, activity, and inducibility of cytochromes P450 in differentiated HepaRG cells. Drug Metab. Dispos. 38, 516-525 (2010).

34. Qiao, L., Sui, J. \& Luo, G. Robust Human and Murine Hepatocyte Culture Models of Hepatitis B Virus Infection and Replication. J. Virol. 92 (2018). 